\title{
Fréquentation des cultures par les abeilles mellifères et sauvages : synthèse des connaissances pour réduire le risque d'intoxication aux pesticides
}

\author{
Axel Decourtye ${ }^{1,2, *}$, Cyril Vidau ${ }^{1}$, Orianne Rollin ${ }^{1}$, Fabrice Requier ${ }^{1}$, Charlotte Rüger ${ }^{1}$, Fabrice Allier ${ }^{1}$, \\ Violette Le Féon ${ }^{1}$, André Kretzschmar ${ }^{1}$, James Devillers ${ }^{1}$, Mickaël Henry ${ }^{1}$ et Jean-François Odoux ${ }^{1}$ \\ ${ }^{1}$ ITSAP-Institut de l'abeille, 228 route de l'aérodrome, Site AgroParc, Domaine INRA Saint Paul, 84914 Avignon cedex 9, France \\ ${ }^{2}$ UMT PrADE, Avignon, France
}

\begin{abstract}
Résumé - En France, la réglementation autorise que les insecticides ou acaricides portant une mention « Abeilles » soient appliqués sur des plantes en fleurs, à condition d'absence de butineuses sur les parcelles à traiter. Mais aujourd'hui, aucun critère n'est disponible pour que l'agriculteur puisse s'assurer de l'absence d'abeilles sur la culture. Pour combler ce manque, le ministère de l'Agriculture a engagé une réflexion sur les facteurs environnementaux et climatiques qui influencent la présence des butineuses sur les parcelles agricoles. Cette revue établit l'inventaire des données disponibles sur les facteurs liés à l'environnement et au climat, qui déterminent l'activité de butinage des abeilles. Des règles de décision basées sur de tels critères apparaissent inappropriées. Seule une pulvérisation une fois le soleil couché réduirait significativement le risque d'exposition des butineuses aux produits.
\end{abstract}

Mots clés : abeilles / pesticides / pollinisation / réglementation / apiculture

\begin{abstract}
Presence of wild and managed bees in the agricultural crops: overview of available data to reduce pesticide risk. In France, a derogation to pesticide regulation allows spraying on blooms with insecticides or acaricides bearing the bee label (mention « Abeilles »), but only when no bees are foraging on crops. Nevertheless, no decision rule is available for farmers to assess bees' absence on the crop. To fill this gap, the Ministry of Agriculture initiated a reflection about environmental and climatic factors influencing the presence of forager bees. This review takes an inventory of data available about foraging activity according to environment or weather. Decision rules based on such criteria appear inappropriate. Only nocturnal sprays of pesticides on crops significantly reduce the risk of bees' exposure to chemicals.
\end{abstract}

Keywords: bees / pesticides / pollination / regulation / beekeeping

La réglementation française définit que les insecticides ou acaricides portant une mention "Abeilles » possèdent une dérogation pour être appliqués sur des plantes en fleurs, à condition d'absence de butineuses sur les parcelles à traiter. Cette réglementation a pour but de protéger l'ensemble des espèces d'abeilles, à savoir l'abeille mellifère élevée par les apiculteurs mais également les abeilles sauvages, environ 1000 espèces en France (Michener, 2007). Une réflexion a été engagée par le ministère chargé de l'agriculture sur les conditions environnementales et climatiques qui garantiraient l'absence d'abeilles sur les parcelles. Cette réflexion impose de répondre à deux questions principales :

- Quels facteurs liés à l'environnement, à la période et au climat, déterminent le mieux l'activité de butinage des abeilles ?

\footnotetext{
* Auteur de correspondance : axel.decourtye@acta.asso.fr
}

- Quelles sont les données disponibles pour classer les cultures en fonction de leur fréquentation par les abeilles ?

Les réponses à ces questions participeront au choix de critères de décision pour l'usage des insecticides ou acaricides portant une mention « Abeilles ».

Cet article a pour objectif de présenter de façon synthétique l'état des connaissances sur les relations entre les abeilles et les cultures, afin d'éclairer les décisions devant encadrer l'emploi des pesticides portant la mention « Abeilles ».

\section{1 «Abeilles » au pluriel}

$\mathrm{Si}$ des diptères (syrphes), des coléoptères ou des lépidoptères (papillons) participent à la pollinisation des plantes à fleurs, les abeilles représentent les insectes 
pollinisateurs les plus importants en nombre d'espèces et en efficacité. En collectant le pollen et le nectar des fleurs pour se nourrir, et nourrir leur descendance, elles transfèrent le pollen de manière passive de fleurs en fleurs. Leur appareil de récolte du pollen (corbeille, brosse) et la forme plumeuse de leurs poils contribuent à cela (Michener, 2007). Les traits biologiques et écologiques du millier d'espèces d'abeilles connues en France sont très divers, en termes d'organisation sociale (mais elles sont solitaires pour la plupart), de spécialisation alimentaire (certaines récoltent leur pollen sur une seule espèce de plante, d'autres sont plus généralistes), de mobilité (de quelques centaines de mètres à plus de $10 \mathrm{~km}$ ) et de types d'habitats (mais la majorité nichent dans le sol). De nombreuses lacunes demeurent sur les modes de vie des espèces sauvages. Alors que l'abeille mellifère est la seule espèce à ce jour considérée dans l'évaluation de la toxicité avant la mise sur le marché des pesticides, de nombreux autres insectes pollinisateurs aux caractéristiques bien différentes peuvent être affectés par l'usage des pesticides. De surcroît, des abeilles sauvages peuvent être plus sensibles aux effets des pesticides que l'abeille mellifère (Rundlöf et al., 2015). Les conditions d'application des pesticides devraient respecter l'ensemble de ce cortège de pollinisateurs.

\section{Exposition des abeilles aux pesticides}

\subsection{Une exposition courante aux pesticides malgré leurs conditions d'emploi restrictives}

S'ajoutant aux travaux réalisés aux États-Unis (Mullin et al., 2010) ou dans le reste de l'Europe (Ghini et al., 2004 ; Bernal et al., 2010), les recherches réalisées en France ont montré que les abeilles mellifères, les miels, les pollens et les cires sont régulièrement contaminés par des résidus de pesticides (Chauzat et al., 2006, 2009; Lambert et al., 2013). Ces travaux décrivent notamment une contamination importante des abeilles, ainsi que du pollen et du miel qu'elles consomment, par des fongicides, des insecticides et des acaricides. Ces derniers peuvent être utilisés par les cultivateurs pour protéger leurs cultures et par les apiculteurs pour lutter contre Varroa, un parasite dangereux pour les colonies. Les résidus d'acaricides synthétiques anti-Varroa pouvant être présents dans les cires des ruches, des méthodes de lutte alternatives se développent (acides organiques, huiles essentielles, sélection génétique...). En consultant les travaux de Lambert et al. (2013) conduits dans l'Ouest de la France en 2009 , on constate que $72 \%$ des échantillons d'abeilles $(n=141), 58 \%$ des pollens $(n=128)$ et $95 \%$ des miels $(n=141)$ collectés contiennent au moins un résidu de pesticide. Plus précisément, ce sont cinq fongicides différents qui sont détectés dans les abeilles. Ils sont au nombre de neuf dans les pollens et dans les miels. Pour les insecticides, 11 résidus différents sont détectés dans les abeilles, alors qu'ils sont respectivement au nombre de dix et de 15 dans les pollens et les miels. Ces résultats montrent que les abeilles sont régulièrement exposées à des insecticides et les fortes concentrations mesurées pour certaines substances (par exemple la cyperméthrine : $48 \mu \mathrm{g} / \mathrm{kg}$ d'abeille) suggèrent une exposition directe des abeilles durant leur activité de butinage.
Tableau 1. Bilan de l'analyse des 165 échantillons de pollen collectés dans cinq régions françaises (Vidau, 2015).

Table 1. Results of chemical analysis of 165 pollen samples collected in five French regions.

Nombre d'échantillons analysés $\quad 165$

Nombre d'échantillons contaminés (\%)

Nombre d'échantillons contaminés par plus de 5 résidus (\%) 25

Nombre de résidus maximum par échantillon $\quad 11$

Nombre total de résidus détectés $\quad 66$

Nombre d'insecticides détectés 23

Nombre de fongicides détectés $\quad 32$

Nombre d'herbicides détectés $\quad 8$

Nombre de régulateurs de croissance détectés 3

\subsection{La co-exposition des abeilles aux pesticides est la règle}

L'amélioration récente de la sensibilité des méthodes d'analyses chimiques multi-résidus rend possible la détection de plusieurs dizaines de substances à des concentrations souvent inférieures à $5 \mu \mathrm{g} / \mathrm{kg}$. Cette avancée a permis de mettre en évidence que les abeilles sont régulièrement exposées de façon concomitante à plusieurs résidus de pesticides. Dans les travaux de Lambert et al. (2013) par exemple, il est décrit qu'en moyenne les abeilles sont contaminées par 1,4 pesticides et que les abeilles les plus contaminées contiennent jusqu'à six résidus de pesticides différents. Aux États-Unis, une contamination importante a été constatée puisque, en moyenne, il a été retrouvé 2,5 résidus de pesticides par échantillon d'abeilles et 25 résidus de pesticides dans l'échantillon le plus contaminé (Mullin et al., 2010). En France, l'alimentation des abeilles est également contaminée par plusieurs résidus comme en témoignent les travaux conduits par l'ITSAP-Institut de l'abeille et les associations régionales de développement apicole : $25 \%$ des pollens collectés par les butineuses sont contaminés par au moins cinq résidus de pesticides (Tab. 1) (Vidau, 2015).

La co-exposition fréquente des abeilles aux pesticides mais aussi à d'autres facteurs de stress (par exemple, agents pathogènes, carences alimentaires) (Goulson et al., 2015) a d'ailleurs conduit l'Agence nationale de sécurité sanitaire (ANSES) à produire un rapport dans lequel elle recommande que la procédure d'évaluation de la toxicité d'un produit phytosanitaire avant sa mise sur le marché intègre des tests pour mesurer l'effet d'une co-exposition chimique à une autre molécule (ANSES, 2015).

\section{Facteurs modulant l'activité de butinage}

La question posée ici est la suivante : quels facteurs liés à l'environnement, à la période et au climat, déterminent l'activité de butinage des abeilles ?

La température ambiante est le facteur météorologique le plus évoqué dans la littérature sur l'activité de butinage. L'humidité relative atmosphérique présente moins d'effet sur l'activité de butinage que la température (Joshi et Joshi, 2010).

Les abeilles ont besoin de maintenir leur température thoracique dans une certaine gamme : $31-32{ }^{\circ} \mathrm{C}$ chez une 
abeille mellifère durant le butinage (Heinrich, 1979). Chaque espèce possèderait ainsi des températures ambiantes seuils (minimale et maximale) au-delà desquelles le coût énergétique nécessaire au maintien de la température corporelle ne pourrait être supporté (Stone, 1994). Selon des auteurs, la masse corporelle est un facteur déterminant de la thermorégulation chez les insectes (Heinrich, 1979 ; Herrera, 1990).

\section{1 À quelle température minimale observe-t-on une activité de butinage?}

Le seuil de température en dessous duquel il n'y a pas d'activité de butinage chez l'abeille mellifère est très variable selon les auteurs : $6^{\circ} \mathrm{C}$ d'après Tan et al. (2012), $7^{\circ} \mathrm{C}$ d'après Heinrich (1979), $9^{\circ} \mathrm{C}$ d'après Burrill et Dietz (1981), $12^{\circ} \mathrm{C}$ d'après Danka et al. (2006), $16^{\circ} \mathrm{C}$ en zone tropicale d'après Joshi et Joshi (2010). Tan et al. (2012) observent une activité de butinage optimale autour de $20^{\circ} \mathrm{C}$. Pour Burrill et Dietz (1981), le seuil de $9{ }^{\circ} \mathrm{C}$ en dessous duquel les abeilles mellifères ne volent pas est indépendant de la luminosité.

Corbet et al. (1993) ont étudié l'effet du climat sur l'activité de butinage de différentes espèces d'abeilles sociales : Apis mellifera et cinq espèces de bourdons (Bombus lapidarius, Bombus terrestris, Bombus lucorum, Bombus pascuorum et Bombus hortorum). En adéquation avec les autres données de la littérature, Corbet et al. (1993) ont montré que la température ambiante et le niveau de radiation étaient positivement corrélés à l'activité de butinage de ces espèces sociales d'abeilles. Ces résultats ont été confirmés au Portugal et au Royaume-Uni sur phacélie et sur des arbustes en fleurs. Les ouvrières d'abeilles mellifères et de $B$. lapidarius commencent à butiner à des températures plus basses que les ouvrières de $B$. terrestris, $B$. lucorum, B. pascuorum et $B$. hortorum. Par rapport à l'abeille mellifère, la plupart des espèces de bourdons sont en effet connues pour avoir une activité de vol à des températures ambiantes inférieures (Corbet et al., 1993 ; Lundberg, 1980 ; Stone et Willmer, 1989).

\subsection{Quelle est la relation entre l'activité de butinage et la température ambiante?}

Burrill et Dietz (1981) ont étudié les effets de plusieurs variables environnementales sur l'activité des butineuses d'abeilles mellifères : ils ont démontré une corrélation de cette activité avec la température et les radiations solaires, deux paramètres évoluant à l'opposé de l'humidité relative et de la pression atmosphérique. Plus la température ambiante augmente, plus le nombre de sorties de la ruche est élevé. L'activité augmente de façon linéaire avec la température entre 13 et $23{ }^{\circ} \mathrm{C}$, indépendamment de la luminosité. Toujours chez cette espèce, d'autres auteurs montrent un effet quadratique de la température (Danka et al., 2006). L'activité (nombre de vols/ unité de temps) augmente avec la température jusqu'à $24{ }^{\circ} \mathrm{C}$. Au-delà de ce seuil, la température a moins d'effet $\left(24-30{ }^{\circ} \mathrm{C}\right)$. Une étude dans des cultures de colza montre qu'entre 27 et $45{ }^{\circ} \mathrm{C}$, la densité d'abeilles mellifères dans les champs diminue avec l'augmentation de la température (Blažyte-Čereškienè et al., 2010). Cet effet inhibiteur des températures élevées serait tout particulièrement marqué chez les butineuses de pollen (Cooper et Schaffer, 1985).
Des inventaires des abeilles, mellifères et sauvages, ont été réalisés sur différents couverts végétaux en fleurs de la zone atelier Plaine-et-Val-de-Sèvre (ZAPVS, Poitou-Charentes). Ils ont été effectués à une température ambiante supérieure ou égale à $16^{\circ} \mathrm{C}$ et à une vitesse de vent inférieure ou égale à $15 \mathrm{~km} / \mathrm{h}$ (Rollin et al., 2013). Les analyses statistiques montrent une influence significative non-linéaire de la température sur la présence et l'abondance des abeilles dans les sites échantillonnés (courbes rouges ; Fig. 1). De plus, l'intensité de cet effet varie en fonction du groupe d'abeilles considéré (abeilles mellifères, bourdons, autres abeilles sauvages) et de la période d'échantillonnage (avril, mai-juin, juillet, septembre). L'abondance des butineuses d'A. mellifera (et de toutes les abeilles) mesurée sur les fleurs diminue aux températures les plus élevées. Cela semble moins évident pour les bourdons et pour les abeilles sauvages. Les résultats d'une étude chez l'espèce sauvage solitaire Anthophora pauperata vont dans le même sens. L'augmentation de la température ambiante jusqu'à $25-30{ }^{\circ} \mathrm{C}$ en milieu de journée n'a pas causé de réduction de l'activité de butinage (Stone et al., 1999). En revanche, une étude sur le butinage de $B$. terrestris réalisée sous serre à fortes températures montre que l'intensité des entrées/sorties de la colonie et l'activité de butinage des ouvrières ont été maximales à $25,7^{\circ} \mathrm{C}$ (durant la matinée). En revanche, à une température moyenne de $32,3{ }^{\circ} \mathrm{C}$, il y a une baisse significative de $70 \%$ de l'activité de butinage et de $40 \%$ du trafic à l'entrée de la colonie (Kwon et Saeed, 2003).

\subsection{L'effet des interactions entre température/période de l'année/heure de la journée}

Des analyses de co-inertie ont été utilisées par Devillers et al. (2004) pour rechercher des liens statistiques entre des données de sorties des ruches enregistrées à l'aide d'un compteur électronique dans la région Rhône-Alpes entre juillet et septembre et la température, l'ensoleillement global, l'humidité, le vent et la pluie. L'existence d'une co-structure entre les activités de sorties des ruches sur 24 heures et la température ou l'ensoleillement global a été clairement établie. Une telle relation n'a pu être montrée à l'échelle du jour pour les autres paramètres abiotiques, même si le vent et la pluie pouvaient avoir une influence ponctuelle très importante.

Dans une zone de grandes cultures (ZAPVS), deux traits de vie ont été enregistrés par l'outil RFID (Radio Frequency IDentification) sur des ouvrières suivies d'avril à août, à savoir le nombre de sorties par jour et la durée de ces sorties. De manière similaire à l'étude précédente, l'activité de vol (reflétée par ces deux traits de vie) est significativement influencée par la température environnante et la période de la saison (mois). L'activité de sortie est corrélée positivement avec la température moyenne quotidienne (Fig. 2). Mais de façon surprenante, les sorties enregistrées en septembre ne présentent pas de corrélation avec la température. En septembre, le nombre de sorties est similaire, qu'il fasse 12 ou $22{ }^{\circ} \mathrm{C}$. Ce mois présente également une moindre influence de la température sur la durée des sorties des ouvrières. Le fait que cette période de l'année soit tout particulièrement dédiée au stockage des réserves alimentaires par les colonies en prévision de l'hivernage pourrait expliquer ce profil d'activité. 

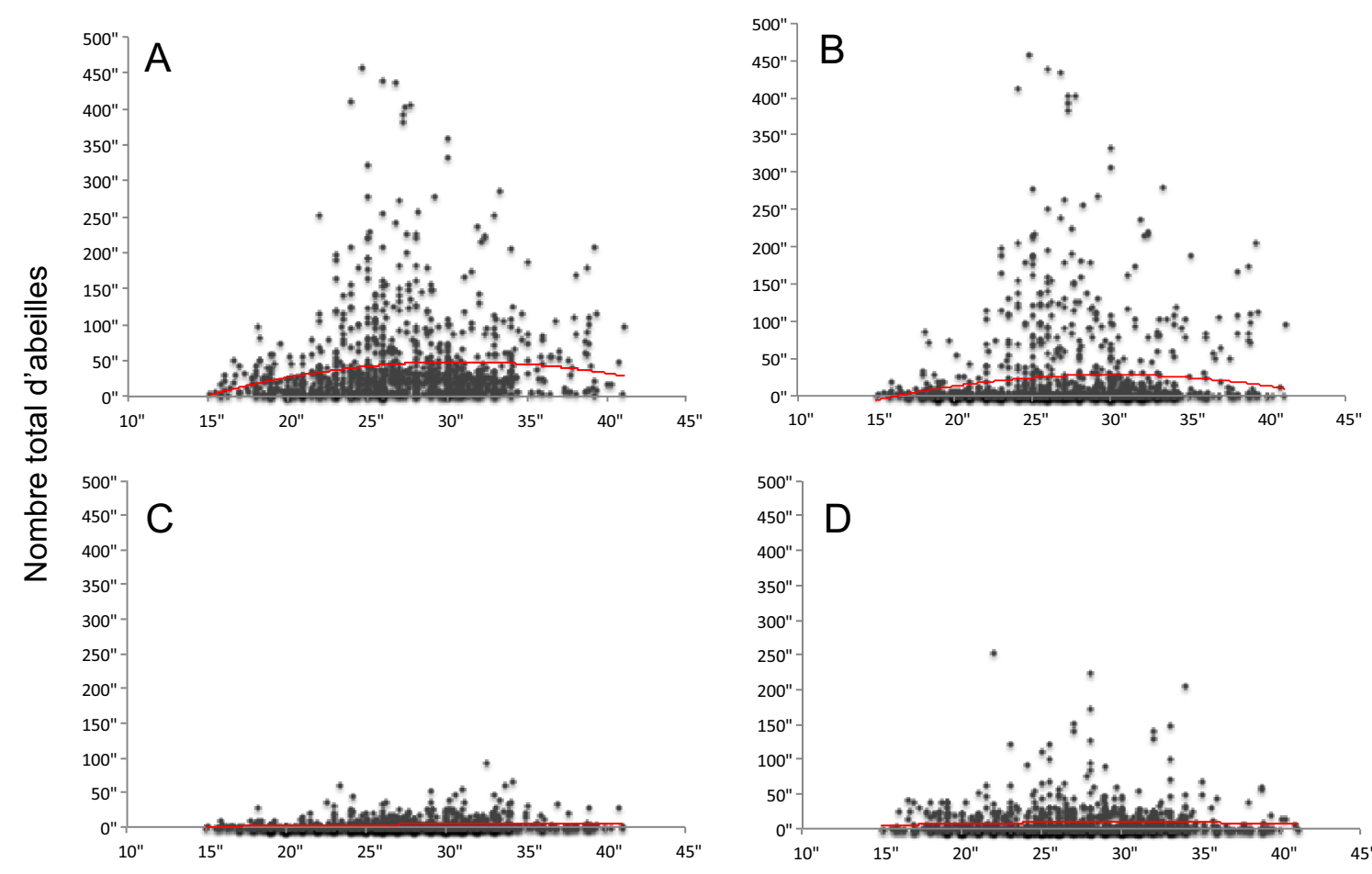

\section{Température $\left({ }^{\circ} \mathrm{C}\right)$}

Fig. 1. Abondance des abeilles mesurée sur des couverts végétaux en fleurs en fonction de la température ambiante. L'axe des $x$ est exprimé en ${ }^{\circ} \mathrm{C}$. Les graphiques montrent l'abondance de toutes les abeilles (A), de l'abeille mellifère (B), des bourdons (C) et des abeilles sauvages autres que bourdons (D). Les points illustrent les données d'abondance et la courbe rouge illustre le résultat du modèle mathématique reliant les données d'abondance à la température (fonction polynomiale ; Rollin, 2013).

Fig. 1. Abundance of bees on blooming plants according to ambient temperature.
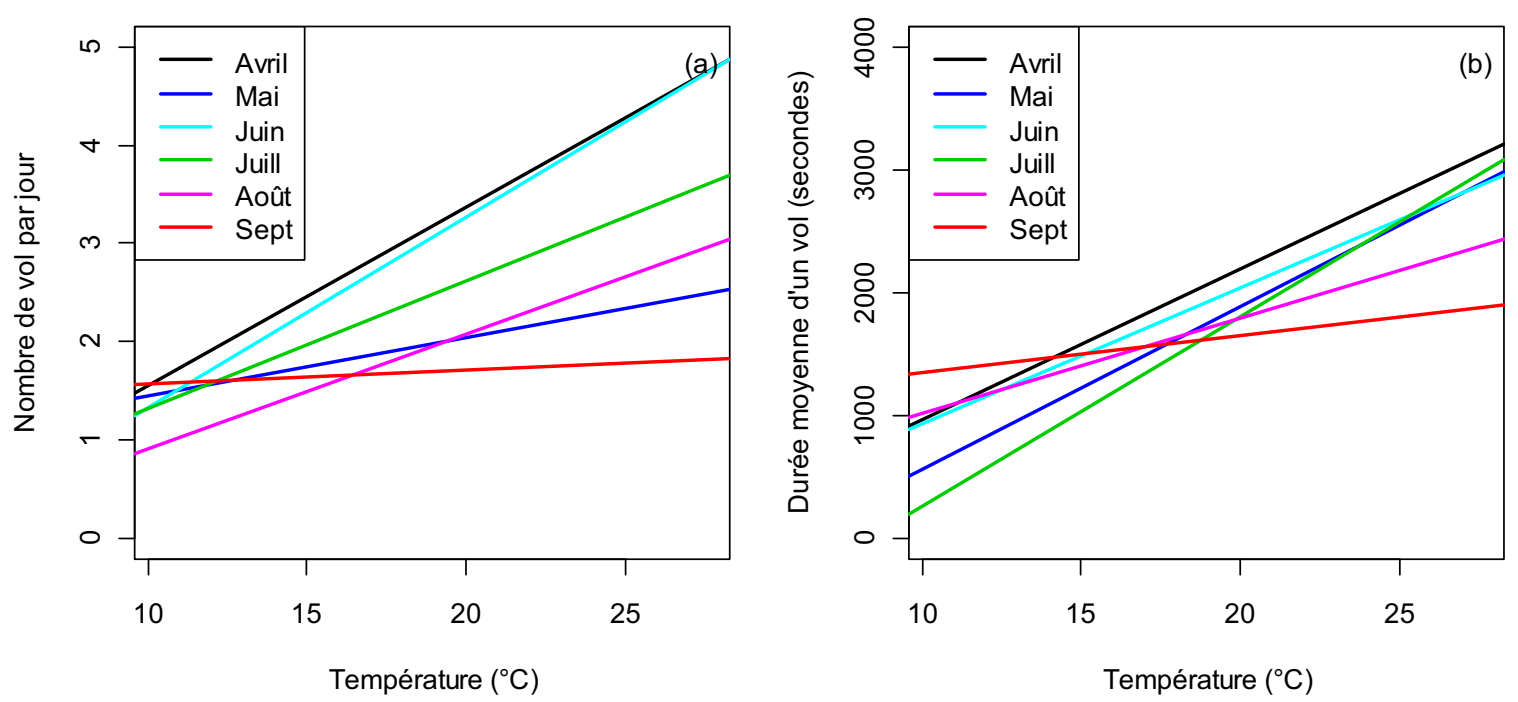

Fig. 2. Prédiction mathématique du nombre de sorties d'abeilles de la ruche en fonction de la température ambiante (moyenne par jour). Les courbes illustrent les résultats de modèles linéaires, tous significatifs, établis à partir d'enregistrements d'ouvrières marquées avec des transpondeurs RFID (Radio Frequency IDentification) ( $n=1330$ abeilles au total ; Requier, 2013). (a) Nombre de sorties par abeille et par jour. (b) Durée moyenne des sorties par abeille et par jour. L'amplitude des températures journalières enregistrée en avril, mai, juin, juillet, août et septembre a été respectivement de 10,2-20,7, 12,9-27,6, 13,7-27,6, 15,1-26,1, 13,3-26,1 et 12,0-22,3 ${ }^{\circ} \mathrm{C}$. Nombre d'abeilles enregistrées par mois $=90-308$.

Fig. 2. Mathematical model that predicts the honeybee exits from the hive according to ambient temperature. 

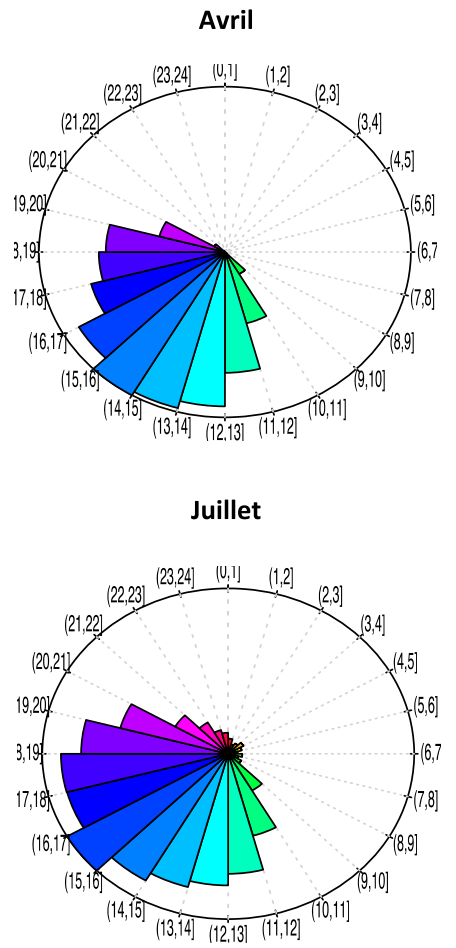

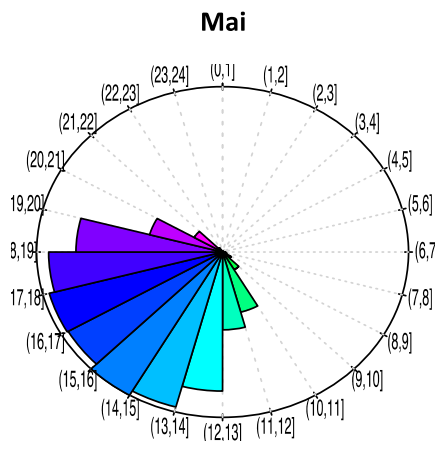

Août

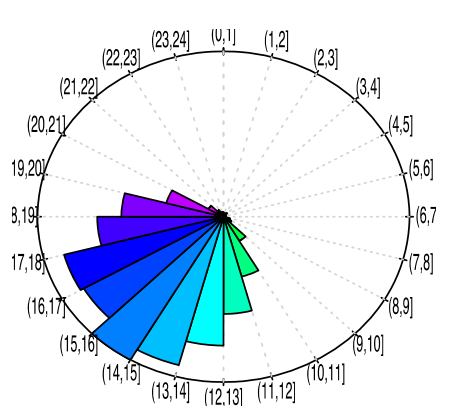

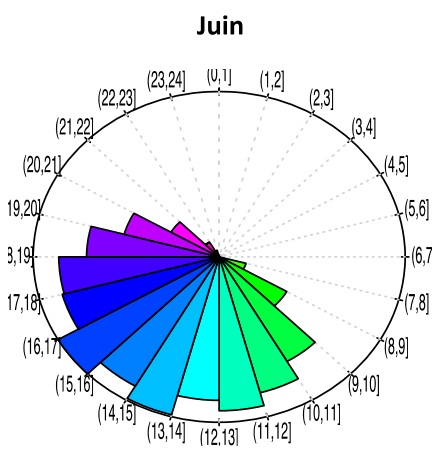

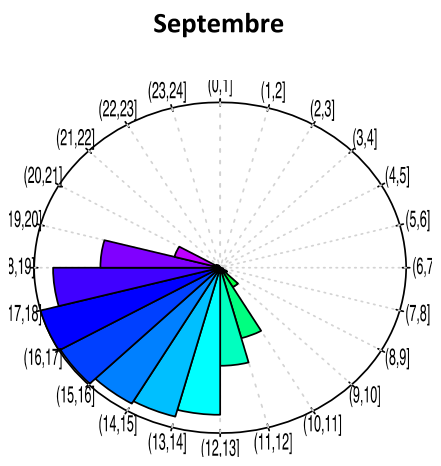

Fig. 3. Plage horaire d'activité des sorties d'abeilles. Les cercles illustrent les 24 heures d'une journée durant laquelle nous avons enregistré les sorties des ouvrières marquées avec des transpondeurs RFID (Radio Frequency IDentification) ( $n=1330$ abeilles au total introduites par cohortes de 90 à 308 individus tous les débuts de mois entre avril et septembre). Les heures sont celles enregistrées par l'ordinateur embarqué du dispositif RFID (Requier, 2013).

Fig. 3. Hours of honeybee exits from the hive.

La plage horaire des sorties des butineuses est variable en fonction du mois (Fig. 3). Cette plage est plus étendue en juin que lors des autres mois. La durée d'ensoleillement par jour doit expliquer en partie ce fait. La moindre quantité de ressources florales disponibles dans le paysage à cette période (Requier, 2013) peut également participer à ce phénomène.

\section{Fréquentation des cultures par les abeilles}

Des inventaires faunistiques réalisés d'avril à août durant trois années (2010-2012), en zone de grandes cultures (ZAPVS) ont consisté à capturer et identifier à l'espèce près de 30000 abeilles butinant sur les fleurs, et ce sur plus de 800 couverts végétaux (Rollin et al., 2013). Ces abeilles ont été classées selon trois groupes: abeilles mellifères, abeilles sauvages et bourdons. Les résultats révèlent de nettes différences dans l'utilisation des ressources fleuries entre les groupes d'abeilles durant la floraison du colza et du tournesol. Les abeilles mellifères, et en moindre mesure les bourdons, préfèrent butiner les fleurs de colza et de tournesol aux fleurs sauvages des prairies et des bordures, alors que c'est l'inverse chez les abeilles sauvages. Les abeilles sauvages ont été observées plus abondement sur les fleurs sauvages des prairies et des bordures plutôt que sur celles du colza ou du tournesol. La diversité en abeilles dans le colza a été quatre fois inférieure à celle mesurée sur une flore herbacée naturelle. Au contraire, les bourdons ont été plus présents sur les fleurs des cultures oléagineuses que sur les autres couverts fleuris, mais de façon moins marquée que l'abeille mellifère.
Les abeilles sauvages utilisent les cultures pour leur ressource alimentaire, comme l'abeille mellifère, mais également pour leur ressource de nidification. En effet, certaines espèces d'abeilles terricoles peuvent nidifier dans le sol au sein des cultures (Julier et Roulston, 2009 ; Shuler et al., 2005). Cette nidification dans les parcelles est à considérer, car environ $80 \%$ des espèces sont terricoles.

Les ressources trophiques se trouvant dans les parcelles cultivées, et pouvant être récoltées par les abeilles butineuses, sont le nectar, le pollen, les gouttes de guttation, le miellat et l'eau. À notre connaissance, l'exploitation par l'abeille de ces trois dernières ressources n'a pas fait suffisamment l'objet de travaux pour les objectiver par rapport aux facteurs de variabilité que pourraient être la plante cultivée, la météorologie, l'heure, la période...

\subsection{Comment classer les plantes cultivées d'après leur fréquentation par les abeilles?}

La base de données de l'ITSAP-Institut de l'abeille rapporte un indice d'attractivité vis-à-vis de l'abeille mellifère, établi à dire d'experts, pour près de 100 cultures ou catégories de cultures. Cette base attribue trois scores pour peu ou pas attractif (1), moyennement attractif(2) ou très attractif (3). Mais cette démarche ne révèle pas la variabilité, pourtant reconnue, de l'intensité de fréquentation d'une culture par les abeilles. D'ailleurs, les données quantitatives disponibles sur le potentiel mellifère par espèce végétale (valeur attendue de la production de miel d'une espèce végétale en kilogrammes par unité de 
surface ; Janssens et al., 2006) traduisent bien cette variabilité. Pour les espèces cultivées, les bases de données connues présentent de grandes variabilités de leurs estimations :

- pour la culture de colza (Brassica napus), le minimum noté est de $67,5 \mathrm{~kg} / \mathrm{ha}$ et le maximum de $325 \mathrm{~kg} / \mathrm{ha}$, soit un facteur de presque 5 entre les deux valeurs estimées ;

- pour le melon (Cucumis melo), plante largement dépendante des pollinisateurs, le minimum observé est de $25 \mathrm{~kg}$ / ha et un maximum de $140 \mathrm{~kg} / \mathrm{ha}$, soit un facteur de plus de 5 entre les deux valeurs estimées ;

- pour le poirier (Pyrus communis), espèce cultivée sur différents territoires (floraison mars-avril), les sources bibliographiques révèlent des potentiels mellifères présentant des écarts d'un facteur 9 selon l'auteur : de 6 à $50 \mathrm{~kg} / \mathrm{ha}$ d'après respectivement Koltowski (2006) et Janssens et al. (2006).

Cette variabilité s'explique par la méthode employée, mais également par l'état de santé et de développement des abeilles, des conditions environnementales, agronomiques (conduites agricoles, variété) et pédo-climatiques. Une autre limite majeure de l'indicateur est qu'il n'intègre pas la fréquentation des plantes par les abeilles pour le pollen.

\subsection{Butinage des plantes adventices des cultures}

Les butineuses d' $A$. mellifera visitent les parcelles de maïs pour le pollen des plantes cultivées (représentant jusqu'à $50 \%$ des apports en pollen d'une colonie lors de la floraison ; Decourtye et al., 2008 ; Pilling et al., 2013 ; Requier, 2013), mais elles y utilisent également des plantes adventices. Par exemple, le pollen de la mercuriale annuelle (Mercurialis annua), une adventice très fréquemment observée dans les parcelles de maïs, est couramment retrouvé dans les pelotes des butineuses en été (jusqu'à plus de $15 \%$ des apports). Mais l'utilisation des plantes adventices comme ressource pollinique en zone de grandes cultures dépasse largement le cas des espèces inféodées au maïs. Cette utilisation est étonnante en termes de diversité de plantes exploitées, de fréquence et de quantité de pollen rapportée à la ruche. Ainsi, 96 pollens différents ont été recensés dans les échantillons de l'observatoire ECOBBE (Odoux et al., 2014), représentant plus de $30 \%$ des apports polliniques lors de la période de floraison du maïs.

Donc le classement des plantes cultivées en fonction de leur probable fréquentation par les abeilles mellifères a ses limites, puisqu'une parcelle d'une plante cultivée ne produisant ni nectar, ni pollen peut accueillir des espèces sauvages attractives. Par exemple, une parcelle d'une céréale à paille présentant peu d'intérêt, sera largement visitée par les butineuses si elle accueille des adventices telles que le bleuet (Centaurea cyanus) ou le coquelicot (Papaver rhoeas L.). Le pollen de coquelicot peut représenter dans une zone de grandes cultures $10 \%$ de la biomasse en pollen récolté par les colonies d'avril à septembre, soit le deuxième pollen le plus récolté après celui du maïs (Requier, 2013). Mais l'importance des ressources offertes par les adventices des cultures n'est pas réservée aux zones céréalières. En juillet 2013, plusieurs échantillonnages polliniques ont été effectués sur un observatoire lors de la miellée de lavande, à l'aide de trappes à pollen $(n=5)$, dans huit ruchers suivis en Drôme provençale. L'analyse palynologique de chacun des échantillons révèle que la plupart des pollens n'appartiennent pas à des plantes cultivées mais à une flore semi-naturelle (ou spontanée) retrouvée dans les surfaces d'interstices. Plus précisément, les espèces du genre Asteraceae (40\%) et les plantains $(15 \%)$ sont les espèces florales les plus utilisées par les abeilles dans un paysage qui est principalement composé de vignobles, de céréales et de lavandes. Cet approvisionnement pollinique s'est avéré contaminé par 40 résidus de pesticides. Ainsi, compte tenu de la nature des résidus de pesticides retrouvés et des calendriers de traitements appliqués au mois de juillet dans ce territoire, ces observations suggèrent que les abeilles sont massivement exposées aux pesticides appliqués sur la vigne et qui contaminent la flore spontanée classiquement retrouvée dans les inter-rangs ou dans les bords de parcelles.

\subsection{Comment se distribuent spatialement les butineuses au sein des parcelles ?}

La répartition spatiale des butineuses au sein des parcelles cultivées est une question couramment posée. Mesurant le butinage des abeilles mellifères selon un gradient de distance à la bordure d'une parcelle de tournesol à son centre, Saez et al. (2012) trouvent une réduction de $25 \%$ de la fréquence de visites sur des capitules entre 1 et $100 \mathrm{~m}$. Sur le maïs, la densité des butineuses par surface est réduit d'un facteur trois entre les premiers rangs et le centre de la parcelle (Thibord et al., 2015). Cela n'est pas retrouvé chez le colza où l'abondance totale des pollinisateurs, celle d'A. mellifera, des syrphes et des bourdons (mais pas pour les abeilles solitaires) est plus importante à l'intérieur des parcelles $(5 \mathrm{~m}$ de la bordure et au centre) qu'en bordure (Fig. 4). La distribution spatiale des butineuses de bourdons privilégiant le centre des parcelles de colza observée en France (zone atelier de PleineFougères, Bretagne) est confirmée par les résultats obtenus au Danemark (Calabuig, 2000) ; dans cette dernière étude, en revanche, les abeilles sauvages solitaires sont plus abondantes en bordure de la parcelle. Ce résultat est confirmé dans une récente étude en France où la distance à la bordure a un effet négatif sur l'abondance d'abeilles sauvages telles que les Andrena et les Nomada dans les cultures de colza (Bailey et al., 2014). Ces auteurs mettent en évidence une influence significative de la distance inter-tégulaire (distance séparant la base des deux ailes des abeilles) sur la répartition spatiale de différentes espèces d'abeilles au sein des parcelles de colza. Cette mesure de trait morphologique reflète la taille des individus et correspond à un estimateur de leur capacité de vol. Les abeilles de grande taille (telles que les bourdons) auront tendance à aller plus loin à l'intérieur des parcelles de colza que les abeilles de plus petite taille (telles que les Andrena et les Nomada).

\section{Conclusion}

En France, une réflexion a été initiée pour identifier des critères de décision qui pourraient être utilisés par les agriculteurs pour pulvériser les insecticides et acaricides portant une mention " Abeilles » afin de réduire le risque d'exposition des abeilles butineuses (consultation publique en 2014). 


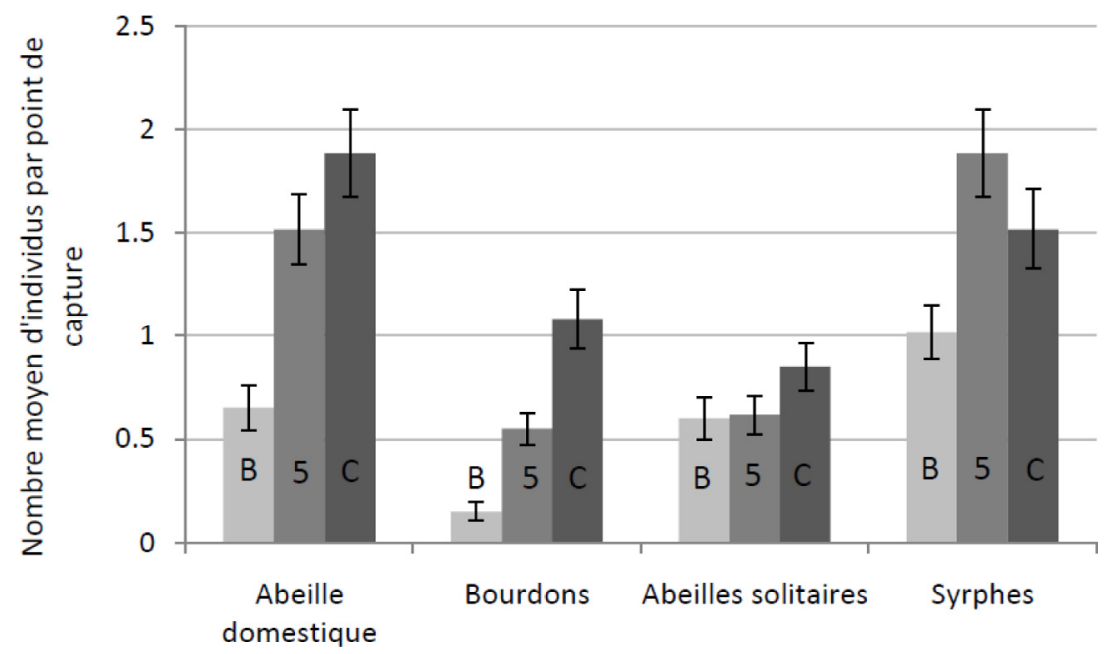

Fig. 4. Nombre moyen de butineuses en fonction des positions dans la parcelle de colza. Les barres représentent l'erreur standard. B : bordure, 5 : à $5 \mathrm{~m}$ de la bordure, $\mathrm{C}$ : centre de la parcelle (méthode décrite dans Le Féon, 2010).

Fig. 4. Average of foraging bees according to position in the oilseed rape crop.

Une option actuellement débattue serait d'aboutir à des règles de décisions différenciées par culture. Pour cela, des données sont disponibles pour attribuer aux plantes cultivées un degré de fréquentation par l'abeille mellifère (score d'attractivité, potentiel mellifère, voire pollinifère). Cette option a des limites pour décliner les recommandations d'usage des pesticides par culture à titre générique sur l'ensemble des conditions, pour les raisons suivantes :

- le degré de fréquentation des cultures n'est pas documenté pour l'ensemble des abeilles sauvages ;

- de multiples facteurs (autres que ceux liés à l'abeille ellemême) vont moduler cette fréquentation par les abeilles (variété, pédo-climat, conduite des cultures, état de santé des abeilles, présence d'autres floraisons, d'autres pollinisateurs ou de bioagresseurs...) ;

- la flore naturelle présente dans les parcelles cultivées ou à proximité de celles-ci représente également une voie d'exposition des abeilles, mellifères ou sauvages, aux pesticides ;

- concernant les abeilles sauvages, les espèces terricoles peuvent nidifier dans les parcelles agricoles ;

- aucune règle, vraie pour l'ensemble des cultures et des espèces d'abeilles, n'est aujourd'hui établie sur la distribution spatiale des butineuses au sein des parcelles. Cela rendrait caduque une règle de décision du traitement qui serait fonction de la surface à traiter (par exemple, « ne pas traiter les $x$ rangs de la culture $y$ en fleurs »).

Une seconde option qui été avancée serait d'employer des critères d'ordre météorologiques (par exemple, « traiter si la température est inférieure à $\left.x^{\circ} \mathrm{C} »\right)$. Si la température reste le paramètre météorologique qui explique une grande part de la variabilité de l'activité de butinage des abeilles, la notion de « températures seuils » au-delà desquelles cette activité serait absente est mise à mal par l'influence significative d'autres facteurs liés aux abeilles elles-mêmes (espèce, santé, cycle de développement) ou liés à la luminosité, au vent, à l'environnement (qualité, quantité et localisation des ressources), à la période de l'année (durée d'ensoleillement) et à l'instant de la journée (e.g., Danka et al., 2006 ; Woyke et al., 2003 ; Burrill et Dietz, 1981). Notre état des connaissances n'est pas suffisant à ce jour pour définir des règles de décision qui combineraient l'ensemble de ces paramètres. Pour ces raisons, des règles de décision basées sur des critères météorologiques nous semblent inappropriés.

En conséquence, seule une pulvérisation une fois le soleil couché réduirait significativement le risque d'exposition des butineuses aux produits phytosanitaires.

Remerciements. Les données publiées par Rollin et al. (2013) et Requier (2013) ont été acquises grâce à l'observatoire ECOBEE dans la zone atelier Plaine-et-Val-de-Sèvre lors du projet « POLINOV : conception et évaluation de systèmes de culture innovants conciliant les enjeux de protection des abeilles et de durabilité de l'agriculture » piloté par l'ACTA et financé dans le cadre d'un appel à projet CASDAR (2009). La base de données des indices d'attractivité des plantes vis-à-vis de l'abeille mellifère a été créée dans le projet « RésApi : mise en place d'un réseau prototype de ruchers ateliers pour expérimenter, se concerter et innover face à l'enjeu des pertes hivernales de colonies » co-piloté par ACTA/ITSAP-Institut de l'abeille et financé dans le cadre d'un appel à projet CASDAR (2011). Le potentiel mellifère des plantes est recensé dans une base de données de l'ITSAP-Institut de l'abeille mise en ligne (http://www.interapi.itsap.asso.fr/) et créée lors du projet INTERAPI ( Influence des CIPAN produisant du nectar et $\mathrm{du}$ pollen en zone de grandes cultures sur la dynamique de colonies d'abeilles mellifères hivernantes $»)$, financé dans le cadre d'un appel à projet CASDAR (2011). Les analyses de résidus de pesticides dans le pollen prélevé en Drôme provençale sont issues du projet intitulé «APIMODEL, modélisation fonctionnelle de l'activité des colonies d'abeilles pour caractériser des seuils de dysfonctionnement à l'échelle du rucher. Généralisation à partir de la miellée sur lavandes ", piloté par l'INRA UR biostatistique et processus spatiaux et financé par le Programme communautaire pour l'apiculture 2013-2016. L'observatoire des résidus de pesticides est financé par FranceAgriMer et le CASDAR. 


\section{Références}

ANSES. 2015. Co-exposition des abeilles aux facteurs de stress. Avis de l'Anses - Rapport d'expertise collective, p. 268. Disponible sur https://www.anses.fr/fr/system/files/SANT2012sa0176Ra.pdf.

Bailey S, Requier F, Nusillard B, Roberts SPM, Potts SG, Bouget C. 2014. Distance from forest edge affects bee pollinators in oilseed rape fields. Ecol. Evol. 4(4): 370-380. doi: 10.1002/ece3.924.

Bernal J, Garrido-Bailón E, Del Nozal MJ, et al. 2010. Overview of pesticide residues in stored pollen and their potential effect on bee colony (Apis mellifera) losses in Spain. J. Econ. Entomol. 103: 1964-1971.

Blažyte-Čereškienė L, Vaitkevičienė G, Venskutonytė S, Būda V. 2010. Honey bee foraging in spring oilseed rape crops under high ambient temperature conditions. Žemdirb. (Agric.) 97: 61-70.

Burrill RM, Dietz A. 1981. The response of honey bees to variations in solar radiation and temperature. Apidologie 12: 319-328.

Calabuig I. 2000. Solitary bees and bumblebees in a Danish agricultural landscape. PhD-Thesis, University of Copenhagen. Disponible sur http://www.zmuc.dk/EntoWeb/staff/ICalabuig/ $\mathrm{PhD}$ thesis Isabel Calabuig.pdf.

Chauzat MP, Faucon JP, Martel AC, Lachaize J, Cougoule N, Aubert M. 2006. A survey of pesticide residues in pollen loads collected by honey bees in France. J. Econ. Entomol. 99: 253-262.

Chauzat MP, Carpentier P, Martel AC, et al. 2009. Influence of pesticide residues on honey bee (Hymenoptera: Apidae) colony health in France. Environ. Entomol. 38: 514-523.

Cooper PD, Schaffer WM. 1985. Temperature regulation of honey bees (Apis mellifera) foraging in the Sonoran desert. J. Exp. Biol. 114: $1-15$.

Corbet SA, Fussell M, Ake R, et al. 1993. Temperature and the pollinating activity of social bees. Ecol. Entomol. 18: 17-30.

Danka RG, Sylvester HA, Boykin D. 2006. Environmental influences on flight activity of USDA-ARS Russian and Italian stocks of honey bees (Hymenoptera: Apidae) during almond pollination. J. Econ. Entomol. 99: 1565-1570.

Decourtye A, Odoux JF, Cluzeau-Moukay S. 2008. L'influence des aménagements floristiques sur les abeilles. Bull. Tech. Apic. 35(3): 114-123.

Devillers J, Doré JC, Tisseur M, Cluzeau S, Maurin G. 2004. Modelling the flight activity of Apis mellifera at the hive entrance. Comput. Electron. Agric. 42: 87-109.

Ghini S, Fernández M, Picó Y, et al. 2004. Occurrence and distribution of pesticides in the province of Bologna, Italy, using honey bees as bioindicators. Arch. Environ. Contam. Toxicol. 47(4): 479-488

Goulson D, Nicholls E, Botías C, Rotheray EL. 2015. Bee declines driven by combined stress from parasites, pesticides, and lack of flowers. Science 26: 1-16.

Heinrich B. 1979. Bumblebee economics. Cambridge, MA: Harvard University Press.

Herrera CM. 1990. Daily patterns of pollinator activity, differential pollinating effectiveness, and floral resource availability, in a summer-flowering Mediterranean shrub. Oiko 58: 277-288.

Janssens X, Bruneau E, Lebrun P. 2006. Prévision des potentialités de production de miel à l'échelle d'un rucher au moyen d'un système d'information géographique. Apidologie 37: 351-365.

Joshi NC, Joshi PC. 2010. Foraging behaviour of Apis spp. on apple flowers in a subtropical environment. N Y Sci J 3, 71-76.

Julier HE, Roulston TH. 2009. Wild bee abundance and pollination service in cultivated pumpkins: farm management, nesting behavior and landscape effects. J. Econ. Entomol. 102: 563-573.

Koltowski Z. 2006. Honey potential of plants growing under Poland condition, Poland: Pulawy.
Kwon YJ, Saeed S. 2003. Effect of temperature on the foraging activity of Bombus terrestris L. (Hymenoptera: Apidae) on greenhouse hot pepper (Capsicum annuum L.). Applied Entomology and Zoology 38: 275-280.

Lambert O, Piroux M, Puyo S, Thorin C, L'Hostis M, Wiest L, et al. 2013. Widespread occurrence of chemical residues in beehive matrices from apiaries located in different landscapes of Western France. PLoS ONE 8(6): e67007. doi: 10.1371/journal.pone.0067007.

Le Féon V, 2010. Insectes pollinisateurs dans les paysages agricoles : approche pluri-échelle du rôle des habitats semi-naturels, des pratiques agricoles et des cultures entomophiles. Thèse, Université de Rennes 1. Disponible sur https://hal.archives-ouvertes.fr/tel00566660/.

Lundberg H. 1980. Effects of weather on foraging flights of bumblebees (Hymenoptera, Apidae) in a subalpine/alpine area. Holarctic Ecol. 3: 104-110.

Michener CD. 2007. The Bees of the World, vol 2nd Revised edition. Hopkins University Press.

Mullin CA, Frazier M, Frazier JL, et al. 2010. High levels of miticides and agrochemicals in North American apiaries: implications for honey bee health. PLoS One 5(3): e9754, doi: 10.1371/journal. pone.0009754.

Odoux JF, Aupinel P, Gateff S, Requier F, Henry M, Bretagnolle V. 2014. ECOBEE: a tool for long-term bee colony monitoring at landscape scale in West European intensive agrosystems. J. Apic. Res. 53(1): 57-66.

Pilling E, Campbell P, Coulson M, Ruddle N, Tornier I. 2013. A fouryear field program investigating long-term effects of repeated exposure of honey bee colonies to flowering crops treated with thiamethoxam. PLoS One 8(10): e77193, doi: 10.1371/journal. pone.0077193.

Requier F. 2013. Dynamique spatio-temporelle des ressources et écologie de l'abeille mellifère en paysage agricole intensif. Thèse, université de Poitiers.

Rollin O. 2013. Étude multi-échelle du patron de diversité des abeilles et utilisation des ressources fleuries dans un agrosystème intensif. Thèse, université d'Avignon et des Pays-du-Vaucluse. Disponible sur https://tel.archives-ouvertes.fr/tel-00993034/document.

Rollin O, Bretagnolle V, Decourtye A, et al. 2013. Differences of floral resource use between honey bees and wild bees in an intensive farming system. Agric. Ecosyst. Environ. 179: 78-86.

Rundlöf M, Andersson GK, Bommarco R, et al. 2015. Seed coating with a neonicotinoid insecticide negatively affects wild bees. Nature 521: 77-80, doi: 10.1038/ nature14420, PMID: 25901681.

Saez A, Sabatino M, Aizen MA. 2012. Interactive effects of large- and small-scale sources of feral honey-bees for sunflower in the Argentine Pampas. PLoS One 7(1): e30968.

Shuler RE, Roulston TH, Farris GE. 2005. Farming practices influence wild pollinator populations on squash and pumpkin. J. Econ. Entomol. 98: 790-795.

Stone GN, Willmer PG. 1989. Warm-up rates and body temperature in bees; the importance of body-size, thermal regime and phylogeny. $J$. Exp. Biol. 147: 303-328.

Stone GN. 1994. Activity patterns of females of the solitary bee Anthophora plumipes in relation to temperature, nectar supplies and body size. Ecol. Entomol. 19: 177-189.

Stone GN, Gilbert F, Willmer P, Potts S, Semida F, Zalat S. 1999. Windows of opportunity and the temporal structuring of foraging activity in a desert solitary bee. Ecol. Entomol. 24: 208-221.

Tan K, Yang S, Wang Z, Radloff SE, Oldroyd BP. 2012. Differences in foraging and broodnest temperature in the honey bees Apis cerana and A. mellifera. Apidologie 43: 618-623.

Thibord JB, Marques M, Dionisi M, Bretagnolle V. 2015. Mais quand donc les abeilles vont dans le colza ? Phytoma 681: 39-43. 
Vidau C. 2015. Exposition des colonies d'abeille aux pesticides. In: Actes des $3^{e}$ Journées de la recherche apicole (Paris).
Woyke J, Wilde J, Wilde M. 2003. Flight activity reaction to temperature changes in Apis dorsata, Apis laboriosa and Apis mellifera. J. Apic. Sci. 47: 73-80.

Citation de l'article: Decourtye A, Vidau C, Rollin O, Requier F, Rüger C, Allier F, Le Féon V, Kretzschmar A, Devillers J, Henry M, Odoux J-F. 2016. Fréquentation des cultures par les abeilles mellifères et sauvages : synthèse des connaissances pour réduire le risque d'intoxication aux pesticides. Cah. Agric. 25: 44001. 